Pulgarín. L., Sandoval, J. y Navarro, P. (2020). Formación contable en Colombia: incidencia del contexto político, económico y social. Contaduría Universidad de Antioquia, 77, 167-190.

Doi: https://doi.org/10.17533/udea.rc.n77a06

\title{
Formación contable en Colombia: incidencia del contexto político, económico y social
}

Laura Natalia Pulgarín García

Lnpulgaring@unal.edu.co

Universidad Nacional de Colombia

orcid: 0000-0003-3186-7689

Julián David Sandoval Alarcón

Julian.sandoval@usantotomas.edu.co

Universidad Santo Tomas

orcid: 0000-0002-0299-4331

Paula Andrea Navarro Pérez

panavarrop@libertadores.edu.co

Fundación Universitaria Los Libertadores orcid: 0000-0001-7253-6646 
Formación contable en Colombia: incidencia del contexto político, económico y social

Resumen: A diferencia de los modelos contables empresariales implementados en Colombia a través de procesos de normalización, la formación contable todavía no está estandarizada e históricamente ha estado supeditada a demandas particulares del contexto donde operan los programas de formación. Consecuentemente, este estudio pretende analizar la formación contable en Colombia a la luz de la influencia que tienen los contextos económico, politico y social en distintos niveles de formación superior y distintas categorías municipales. Para ello, se utiliza una metodología descriptiva y analítica, acompañada de un muestreo aleatorio simple que permita contrastar los programas de formación profesional, técnica y tecnológica en el área contable a la luz de la influencia que ejercen sus entornos. Se encontró que, los programas profesionales tienen mayor influencia del contexto en comparación a los técnicos y tecnológicos; además, de dicho contexto el aspecto que más influye es el político y el que menos es el social.

Palabras clave: Educación contable, contexto, politica, economía, sociedad.

Accounting training in Colombia: incidence of the political, economic, and social context

Abstract: Unlike the business accounting models implemented in Colombia through normalization processes, accounting training has not been standardized yet and has historically been subjected to particular demands from the context where the educational programs operate. Consequently, this study seeks to analyze accounting training in Colombia regarding the influence of the economic, political and social contexts on the different levels of higher education and diverse municipality categories. To do so, a descriptive and analytical methodology is used, together with a simple random sampling allowing to contrast training programs at university, technical and associate degree levels in the accounting area regarding the influence exerted by their environments. It was found that university programs have greater influence from the context as compared to technical and associate degree programs; besides, the most influential aspect from said context is the political one, while the social aspect is the least influential.

Keywords: Accounting education, context, politics, economy, society.

Formação contábil na colômbia: incidência do contexto político, econômico e social

Resumo: Ao contrário dos modelos contábeis empresariais implementados na Colômbia por meio de processos de normalização, a formação contábil ainda não está padronizada e historicamente tem dependido de demandas particulares do contexto onde operam os programas de formação. Portanto, este estudo tem como objetivo analisar a formação contábil na Colômbia à luz da influência que têm os contextos econômico, político e social em diversos níveis de formação superior e diferentes categorias municipais. Para tal fim, utiliza-se uma metodologia descritiva e analitica, acompanhada de uma amostragem aleatória simples que permita cotejar os programas de formação profissional, técnica e tecnológica na área contábil à luz da influência que exercem os seus entornos. Encontrou-se que os programas profissionais têm maior influência do contexto em comparação aos técnicos e tecnológicos; além disso, o aspecto que mais influi é o político e o que menos influi é o social.

Palavras chave: Educação contábil, contexto, política, economia, sociedade.

Formation comptable en colombie: l'incidence des contextes politique, économique et social

Résumé: Contrairemente aux modèles de comptabilité d'entreprise mis en ouvre en Colombie au moyen de processus de normalisation, la formation comptable n'est pas encore normalisée et a toujours été soumise à des exigences particulières du contexte dans lequel opèrent les programmes de formation. En conséquence, cette étude vise à analyser la formation comptable en Colombie à la lumière de l'influence des contextes économique, politique et social à différents niveaux de formation supérieure et dans différentes catégories municipales. Pour ce faire, une méthodologie descriptive et analytique a été utilisée, accompagnée d'un échantillonnage aléatoire simple permettant de comparer les programmes de formation professionnelle, technique et technologique dans le domaine comptable à la lumière de l'influence exercée par leur environnement. Il a été constaté que les programmes professionnels ont une plus grande influence du contexte par rapport aux programmes techniques et technologiques, et que en plus du contexte, l'aspect politique est celui qui influe le plus, tandis que l'aspect social a le moins d'influence.

Mots clés: Éducation comptable, contexte, politique, économie, société.

JEL Classification: $I 2, M 4, M 0$ 
Cont. udea (julio-diciembre), pp. 167-190. (C) Universidad de Antioquia-2020.

\title{
Formación contable en Colombia: incidencia del contexto político, económico y social
}

\author{
Laura Natalia Pulgarín García, Julián David Sandoval Alarcón \\ y Paula Andrea Navarro Pérez.
}

https://doi.org/10.17533/udea.rc.n77a06

Primera versión recibida en junio de 2020 - Versión final aceptada en octubre de 2020

\section{Introducción}

D esde los inicios de la contaduría pública como parte de la formación universitaria en Colombia se refleja una clara injerencia del contexto global y los intereses de las instituciones económicas mediados por las políticas estatales (León, 2008), lo cual ha incidido en la estructura que sustenta la formación del contable. Dicha estructura se ha orientado a una construcción curricular que dé respuesta mayoritariamente al cumplimiento de los requerimientos administrativos formalizados por parte del gobierno, específicamente el Ministerio de Educación Nacional (MEN), dejando a un lado las condiciones sociales y culturales de las comunidades donde hacen presencia las instituciones de educación superior (Barragán, 2008). En otras palabras, las sociedades presentan a nivel general un desplazamiento en las lógicas que soportan los sistemas de producción, culturales, financieros y de información, mediante un proceso de modernización en los diferentes saberes epistemológicos al igual que en la praxis; no obstante, dicha transformación ha traído consigo una mirada parcial y poco transversal (Mella, 2003).

Consecuentemente, la disciplina contable se encuentra a nivel general en una dicotomía provista por su componente normativo, con tintes de mecanicismo, y su necesidad interna de mejorar su capacidad de análisis sobre las realidades donde esta se encuentra (Aguilera, 2014). En este sentido, los procesos de formación en contabilidad han sido estudiados desde perspectivas éticas (Colmenares, Valderrama, Perdomo y Ramírez, 2016), teorías de la educación (Loaiza, 2013), características de los estudiantes (Patiño y Santos, 2009; Sebayang y Muda, 2020), formación de capacidades (Del Cortea y Martínez, 
2018; Rangland y Ramachandran, 2014; Setianto y Harahap, 2017; Valero, Patiño y Duque, 2013) y estudios de las instituciones educativas y su oferta académica (Navarro, Pulgarín y Sandoval, 2019; Rojas y Zapata, 2011; Rojas y Sánchez, 2016; Valderrama et al., 2009). Resaltando la ausencia de un estudio que analice de forma integral las relaciones entre la formación del contable en todos los niveles de enseñanza superior con las condiciones fundamentales de sus entornos intrínsecos.

En concordancia con lo anterior, el presente trabajo tiene por objetivo analizar la formación de la disciplina contable en Colombia, bajo su contexto económico, político y social, a través de una revisión de los planes de estudio, perfiles de ingreso, perfiles de egreso, misión y visión de los programas de educación superior en los niveles de técnico, tecnólogo y profesional, para contrastar los contenidos institucionales con los entornos previamente mencionados. En este sentido se establecen categorías sobre los requerimientos del entorno económico, el entorno político y las necesidades sociales, igualmente se utiliza la clasificación de los municipios colombianos, provista por la Contaduría General de Nación, para la vigencia 2020, obteniendo un total de 100 programas reconocidos por el MEN distribuidos en 285 municipios.

Dicho lo anterior, la primera parte del trabajo hace una distinción entre la formación técnica, tecnológica y profesional, y un abordaje conceptual sobre las dimensiones económicas, políticas y sociales de mayor relevancia para el desarrollo de la configuración de la enseñanza de la profesión contable, estableciendo así, cinco categorías de estudio por cada una de las dimensiones. Posteriormente, se coteja con la información recolectada de los programas contables en los tres niveles de enseñanza objeto de estudio, identificando si existía evidencia de la presencia de cada una de las quince (15) categorías de estudio. Finalmente, se plantea una discusión sobre la influencia de cada aspecto contextual en la formación contable y las posibles implicaciones que esto tiene para la disciplina.

\section{Distinción entre formación técnica, tecnológica y profesional}

En Colombia, según la Ley 30 de 1992, los campos de acción de la educación superior son el de la técnica, la tecnología, la ciencia, las humanidades, el arte y la filosofía (Congreso de la República de Colombia, 1992), y dichos campos se agrupan en tres niveles de formación: Técnico Profesional, Tecnológico y Profesional.

Asimismo, de acuerdo con la Ley 749 de 2002, las instituciones técnicas profesionales se caracterizan por su enfoque en el conocimiento y el trabajo de carácter técnico... 
(...) fundamentadas en la naturaleza de un saber, cuya formación debe garantizar

la interacción de lo intelectual con lo instrumental, lo operacional y el saber técnico

(...) en las áreas de las ingenierías, la tecnología de la información y la administración.

(Congreso de la República de Colombia, 2002, art. 2)

Los conocimientos de carácter técnico se relacionan con las competencias, el desarrollo intelectual, aptitudes, habilidades y destrezas necesarias para el desempeño laboral en una actividad, en áreas específicas de los sectores productivo y de servicios.

Por su parte, las instituciones tecnológicas se centran en los conocimientos y profesiones de carácter tecnológico, con fundamentación científica e investigativa, destinadas al igual que para el nivel técnico, a las áreas de las ingenierías, la tecnología de la información y la administración. Este nivel se enfoca en los conocimientos científicos y en la comprensión teórica contribuyendo a la formación de...

(...) un pensamiento innovador e inteligente, con capacidad de diseñar, construir, ejecutar, controlar, transformar y operar los medios y procesos que han de favorecer la acción del hombre en la solución de problemas que demandan los sectores productivos y de servicios. (Congreso de la República de Colombia, 2002, art. 3)

En cuanto al nivel profesional, este se entiende como un tercer ciclo de formación que complementa el nivel tecnológico con la fundamentación teórica y la propuesta metodológica de la profesión, e implica el dominio de conocimientos científicos y técnicos (Congreso de la República de Colombia, 2002).

\section{Contexto de la formación contable en Colombia}

La contabilidad como área de conocimiento tiene su origen y desarrollo en las actividades económicas y sociales de las comunidades. Por ello, tiene sentido que las estructuras de creación y promulgación de estándares que se dan en esta disciplina estén en conjunción con las necesidades del entorno particular donde se desenvuelve, entendiendo a dicho entorno como la estructura mayor que le da soporte y la moldea (León, 2009), denominada por algunos autores como el sistema contable (Jarne, 1997; Gómez, 2007).

No obstante, la lógica financiera ha permeado la esfera contable desde hace ya varias décadas, promulgando un modelo de estandarización de la disciplina representado en estándares internacionales de información financiera (IFRS), de auditoría (ISA), de contabilidad para el sector público (IPSAS) e inclusive de formación (IES). Si bien ya varios países a nivel global han migrado a IFRS, ISA e IPSAS a través de procesos de convergencia y armonización, la difusión de los estándares de formación no ha sido tan vertiginosa a nivel global.

Particularmente en Colombia, con la Ley 1314 del 2009 se dio inicio al proceso de convergencia a IFRS e ISA para el sector privado (Congreso de la República de Colombia, 2009) y a través de las Resoluciones 354 del 
Pulgarín. L., Sandoval, J. y Navarro, P. Formación contable en Colombia...

2007, 628 del 2015, 037 del 2017, 414 del 2014, 533 del 2015 y 461 del 2017 de la Contaduría General de la Nación (CGN), se incorporó un proceso de armonización para el sector público hacia IPSAS e IFRS (CGN, 2007; CGN, 2014; CGN, 2015; CGN, 2017a; CGN, 2017b). A pesar de ello, en materia de formación todavía no se han adoptado en el país medidas que propendan por estandarizar los procesos de creación y emisión de currículos para los programas de Contaduría Pública a nivel de educación superior; si bien se han generado algunas condiciones mínimas de calidad y ofrecimiento por parte del MEN, existe todavía cierta potestad institucional sobre el establecimiento de los currículos. La falta de difusión de las IES, por lo menos en Colombia, se ha debido principalmente a los debates generados en torno a "la adaptación de tales normas en el ámbito nacional (...) y su carácter profesionalizante, supeditado a las demandas del mercado laboral” (López, 2013, p. 241).

En este sentido, el MEN se ha limitado a determinar unos lineamientos básicos en torno a los aspectos curriculares que deben contener los programas de Contaduría Pública a nivel profesional a través de la Resolución 3459 de 2003, donde señala que el plan de estudios comprende, como mínimo, cursos en las áreas de formación básica, sociohumanística y profesional, contemplando dentro de esta última los componentes Ciencias contables y financieras, Formación organizacional, Información y Regulación (MEN, 2003a). En cuanto al área de formación socio-humanística, incluye aquellos "saberes y prácticas que complementan la formación integral del Contador Público, orientados a proporcionar una visión holística del ejercicio profesional que facilita el diálogo interdisciplinario y el trabajo con profesionales de otras disciplinas y profesiones" (MEN, 2003a, art. 2) y el desarrollo de ética profesional.

En cuanto a los programas del nivel técnico y tecnológico, la Resolución 3462 de 2003, por la cual se definen las características específicas de calidad para los programas de formación hasta el nivel profesional por ciclos propedéuticos en las áreas de las Ingeniería, Tecnología de la Información y Administración, el programa curricular y el plan de estudios en contabilidad, como parte del área de administración, está integrado por las áreas de formación básica, profesional, socio-humanística, de énfasis y de comunicación. Dentro del área de formación profesional se incluyen los componentes de la economía, las finanzas, administración y organizaciones, producción y operaciones, mercadeo, informática y gerencia personal; mientras que el área de formación socio-humanística se encuentra integrada por saberes y prácticas como complemento, para una formación axiológica, sociológica, cultural y en humanidades, orientada hacia el conocimiento de los contextos sociales y culturales de las organizaciones (MEN, 2003b, art. 21 y 23). A pesar de que las áreas y los componentes de estas son los mismos, el MEN realiza precisiones en la descripción de cada uno de ellos para resaltar la diferencia entre 
ambos niveles de educación superior de manera que para el técnico se busca desarrollar competencias necesarias para la solución de problemas puntuales y para garantizar la interacción de lo intelectual con lo instrumental, mientras que para el tecnológico, las competencias a desarrollar deben contribuir a la solución de problemas en el área a través de la planificación, la organización, el control y la dirección de proyectos y actividades (MEN, 2003b, art. 22 y 24).

Paralelamente, a través de los años los procesos de creación y emisión de currículos para los programas del área contable en el país han estado sujetos a variables del entorno, principalmente aquellas que le dan un carácter interdisciplinar a la contabilidad, entendiendo dicha interdisciplinariedad como:

La posibilidad de relación entre varias disciplinas o ramas del conocimiento que enriquecen la perspectiva del escenario, creando posibilidades y abriendo horizontes más amplios a la realidad que se quiere dar a conocer; esta permite reducir el impacto de la especialización y la fragmentación del conocimiento. (Moreno y Duque, 2016, p. 28)

De acuerdo con Palacio y Navas (2011) "en su historia la contabilidad más que otras disciplinas surge de la necesidad de hacer elaboraciones técnicas inicialmente, y posteriormente teóricas de acuerdo a contextos sociales, económicos y políticos definidos" (p. 183). En el país, la formación contable no es ajena a estos tres factores. En principio, el factor preponderante es el tejido económico y empresarial de la nación, expresado en la demanda laboral que tienen las empresas e instituciones sobre los egresados. Adicionalmente, la influencia del sistema político es también importante, dado que dicho sistema moldea no solo las normativas que aplican al diseño del currículo sino también aquellas que se dirigen al ejercicio de la profesión contable. Por último, la sociedad como tal, a través de los individuos y las colectividades, también ejerce unas demandas relevantes para los procesos de formación contable.

\section{III.I. Contexto económico}

La economía del país plantea un escenario en el que se le exige a las instituciones de educación superior formar individuos capaces de fortalecer el tejido empresarial dado un macro-contexto (Casanova, 2003). Específicamente, se dice que este fortalecimiento se da en la medida en que se incremente la productividad y la competitividad de las empresas donde los individuos formados actúan.

La demanda del contexto en términos económicos para quienes ejercen la profesión contable se ve reflejada en dos ámbitos principales. Por un lado, se requiere que haya conocimientos de los mercados internacionales, de globalización, de flujo de capitales y de estándares internacionales para estos ambientes; por otro lado, se requiere también una visión local, que permita conocer el tamaño de las empresas que componen el entorno y el sector en el que operan. Estos requerimientos se materializan en modelos de formación 
Pulgarín. L., Sandoval, J. y Navarro, P. Formación contable en Colombia...

basados en habilidades y destrezas dirigidos principalmente hacia el mundo de los negocios (López, 2013).

Particularmente, Colombia se caracteriza por ser una economía con varios tratados de comercio vigentes con distintos países a nivel global. Desde la década de los 90, las reformas estructurales que se han hecho en el país han propendido por internacionalizar la economía y abrir los mercados de capitales (Bonilla, 2011). En la actualidad, los países con los que más transacciones se llevan a cabo son Estados Unidos, China, Panamá, Países Bajos, México, Brasil y Alemania, exportando productos como el petróleo, combustibles, café, aceites y minerales, e importando tecnologías, automóviles, medicamentos y aeronaves (OEC, 2018). Además, se cuenta con alta presencia de multinacionales extranjeras que operan en el territorio nacional y también algunas de las empresas colombianas operan en territorio internacional.

En el contexto local, las Mipymes representan 96\% de la economía colombiana, operando principalmente en los sectores de servicios, comercio y manufactura (ACOPI, 2019). En términos regionales, Bogotá es la ciudad que cuenta con mayores tasas de creación empresarial y participación de las mismas en la economía nacional, acompañada de departamentos como Antioquia, Valle del Cauca, Cundinamarca, Atlántico y Santander; sin embargo, en los últimos años Chocó, La Guajira, Sucre y Amazonas han tenido altos índices de crecimiento respecto a dichas tasas de creación (Confecámaras, 2018).

\section{III.II. Contexto político}

Frente al escenario de las políticas públicas, Peralta y Vasco (1998) refiriéndose a los factores que impulsan el desarrollo del país exponen como claro ejemplo la constitución de 1991, donde expresan que "las verdaderas reformas que requería el país fueron sustituidas por modificaciones accesorias y formales del sistema político, sin que se tocara nunca el problema de fondo de la distribución del poder y su interrelación con la estructura económica" (p. 1), se infiere que el estudio del campo reglamentario es fundamental para el entendimiento y desarrollo de cualquier tipo de actividad.

Por otra parte, en la Cumbre Mundial sobre la Sociedad de la Información (2005) se resalta la importancia del crecimiento y fortalecimiento de las tecnologías de la información y la comunicación bajo los principios de la igualdad y asociación, debido a la creciente tendencia de la transformación de la información y el conocimiento en recursos privados susceptibles de ser mercantilizados, sin atender a su esencia fundamental de componente del desarrollo social. Igualmente, Jokinen (2000) argumenta que la implementación de una "sociedad de información" se relaciona como resultado de la preocupación estatal sobre la creciente competencia en un mercado globalizado, además de incentivar una mejoría en la competitividad y 
productividad del sector público y privado para mantener un bienestar general en la población.

De igual manera, para Gómez (2008), uno de los problemas de la sociedad posindustrial es la ausencia de políticas enfocadas en la preservación de la vida en el planeta a raíz de la intervención humana, por ello, es fundamental fortalecer el marco regulatorio dirigido a las responsabilidades extrahumanas sobre el abuso de la naturaleza e implementar esquemas ideológicos tales como la ecología política, ética política y el humanismo ecológico.

De modo semejante, el gobierno colombiano mediante la Ley 1951 de 2019 crea el Ministerio de Ciencia, Tecnología e Innovación, constituyendo un ente rector que promueva el conocimiento científico y tecnológico, establezca estrategias de transferencia y apropiación social de la ciencia, la tecnología, innovación y emprendimiento, todo esto mediante el establecimiento de políticas públicas. Anterior a la creación del ministerio, el sistema de ciencia, tecnología e innovación se regía por la Ley 1286 de 2009, donde se implementaron estrategias para acercar el sector productivo al aparato científico nacional e internacional, con el fin en generar resultados para la sociedad basados en las capacidades de Investigación y Desarrollo (I+D) del país (Departamento Administrativo de Ciencia, Tecnología e Innovación Colciencias, 2018).

Por último, es importante resaltar que ante los crecientes cambios en las formas de administración estatal este campo de estudio ha tenido que evolucionar con un rango de acción tan diverso que se ha tenido que subdividir o especializarse en saberes particulares, por lo que se apoya de otras disciplinas, ejerciendo así un trabajo multidisciplinario y pluri-metodológico (Martínez, 2009). En efecto, la disciplina contable no es ajena a contribuir sobre la consecución de los objetivos de la administración pública, más aún, siendo parte de las ciencias sociales ayuda a nutrir las teorías de la sociedad y el Estado mediante el fortalecimiento del análisis, la interpretación y la argumentación de los sistemas económicos, políticos y sociales (Aguilera, 2014).

\section{III.III. Contexto social}

Referente a la influencia de los entornos en la composición de los espacios de formación profesional, es fundamental comprender que estos últimos son construcciones colectivas con base en las necesidades sociales o estatales. Según Peralta y Vasco (1998), uno de los principales problemas de los cuales adolece Colombia es la distribución del poder, entendiéndose esta como la forma mediante la cual existe y se utiliza la influencia sectorial para la consecución de intereses específicos. Dicha distribución, evidencia una excesiva influencia de grupos privados y sectoriales, reflejando así la incapacidad del Estado de satisfacer el interés general. 
Pulgarín. L., Sandoval, J. y Navarro, P. Formación contable en Colombia...

Por otra parte, Gómez (2008) discute sobre dos problemas centrales dentro de la sociedad globalizada, los cuales afectan principalmente al comercio y a la sustentabilidad de la vida en el planeta, teniendo en cuenta las brechas que pueden surgir dado el progreso tecnológico de la humanidad, resaltando que las necesidades deben ser abordadas bajo un enfoque de sociedad globalizada.

De acuerdo con Tejada (2014), la construcción de la sociedad civil es una relación de tensiones entre el Estado, la esfera económica y la política, las cuales, mediante un proceso histórico, dictatorial y clientelar, consiguen la obtención de libertades civiles, sin que se pueda afirmar que existe un contenido ideológico único dentro de la colectividad; por el contrario, existe una persistencia de disputas y poderes que permite crear nuevas identidades.

Ahora bien, en las comunidades, entendidas en un sentido amplio, existen discordias basadas en segmentaciones y posturas ideológicas, las cuales requieren un abordaje estratégico y multidisciplinario para contribuir a la cohesión social (Yassim, 2019). Así pues, el estudio, ejercicio y práctica de la disciplina contable es un claro ejemplo al reflejar el desarrollo y apoyo mutuo en disciplinas como la economía y las finanzas, "por ello, pretender explicar y entender el desarrollo de las diferentes disciplinas, exige estudiar el entramado social, en que van surgiendo y consolidándose." (Ortiz, 2009, p. 182).

Finalmente, un aspecto transversal a trabajar en los colectivos sociales es la ética, debido a su aporte de valores normativos promovido mediante principios y prácticas sobre el uso de concepciones como la imparcialidad y la igualdad, permitiendo conformar una conciencia individual, aún dentro de la colectividad e igualmente en las relaciones con otros individuos o comunidades (Uvalle, 2014).

\section{Diseño metodológico}

Para este trabajo, se utilizó una metodología de análisis mixto, combinando criterios cuantitativos y cualitativos. En principio, se llevó a cabo una revisión literaria tradicional para establecer cómo factores contextuales en el plano económico, político y social, influencian los procesos de diseño y emisión de planes de estudio para los programas de formación superior en el área contable. Para esta revisión, se hizo uso del motor de búsqueda Google Scholar junto con bases de datos académicas como Scopus, Science Direct, Redalyc, Dialnet y Scielo. Se utilizaron además palabras clave como formación contable y educación contable, en conjunción con contexto económico, contexto político y contexto social. Asimismo, se incluyeron en la revisión fuentes de información institucionales y gubernamentales que permitieran delimitar el contexto educativo colombiano.

Con base en dicha revisión, se planteó el perfil de Colombia respecto a cada uno de esos factores para así, determinar qué variables de cada componente se espera tendría un currículo si fuese influenciado por cada uno. Dichas variables se resumen en la Tabla 1. 
Tabla 1: Variables contextuales por componente que influencian la formación

\begin{tabular}{|c|c|c|}
\hline Económicas & & \\
\hline $\begin{array}{l}\text { Macro-contexto (internacionalización) } \\
\text { 1. IFRS: Transversalidad del uso de } \\
\text { norma internacional en el currículo, } \\
\text { existencia de al menos una asignatura } \\
\text { de contabilidad internacional o } \\
\text { descripción en el perfil del egresado en } \\
\text { relación con Normas Internacionales de } \\
\text { Contabilidad. } \\
\text { 2. Globalización, libre mercado y } \\
\text { comercio exterior: Existencia de } \\
\text { asignaturas relacionadas con economía, } \\
\text { comercio, negocios y mercados a nivel } \\
\text { internacional. } \\
\text { Contexto local } \\
\text { 3. Tejido empresarial y tamaño } \\
\text { empresarial: Asignaturas relacionadas } \\
\text { con creación de empresas y } \\
\text { emprendimiento en el país. } \\
\text { 4. Sectores y actividades económicas } \\
\text { regionales: Existencia de asignaturas } \\
\text { con enfoque contable hacia un sector } \\
\text { específico en el que se desenvuelve } \\
\text { la comunidad, relacionado con la } \\
\text { actividad económica generadora de } \\
\text { ingresos de la región o con un enfoque } \\
\text { especial determinado por la institución } \\
\text { educativa. } \\
5 \text {. Economía nacional: } \\
\text { Asignaturas cuya estructura se relacione } \\
\text { con coyuntura económica del país, } \\
\text { que contemple un componente crítico } \\
\text { respecto a la economía colombiana o }\end{array}$ & $\begin{array}{l}\text { ncia de derecho en la } \\
\text { ón: Asignaturas con } \\
\text { ente regulatorio, ya } \\
\text { hercial, constitucional, } \\
\text { l, entre otros. } \\
\text { tigación e innovación: } \\
\text { n en el currículo de } \\
\text { uras como metodología } \\
\text { vestigación y } \\
\text { ios de investigación } \\
\text { pción del perfil del } \\
\text { the y egresado en } \\
\text { la investigación. } \\
\text { blogías de la } \\
\text { ción y Comunicación: } \\
\text { herramientas } \\
\text { ticas y tecnológicas en } \\
\text { ación del estudiante } \\
\text { las en el área contable } \\
\text { istrativa. } \\
\text { oambiente y calidad } \\
\text { rocesos: Asignaturas } \\
\text { ladas con el papel de } \\
\text { cias económicas en la } \\
\text { ión y conservación del } \\
\text { ambiente. } \\
\text { r público: Asignaturas } \\
\text { ladas con finanzas } \\
\text { puesto público, } \\
\text { Ira política y } \\
\text { ica estatal. }\end{array}$ & $\begin{array}{l}\text { 1. Prácticas sociales: } \\
\text { Asignaturas relacionadas con } \\
\text { la contabilidad como parte } \\
\text { de actividades de tipo social } \\
\text { o consultorios contables. } \\
\text { 2. Responsabilidad social } \\
\text { Empresarial: Asignaturas que } \\
\text { relacionen a la contabilidad } \\
\text { con responsabilidad social, } \\
\text { sostenibilidad y reportes } \\
\text { integrados. } \\
\text { 3. Identidad y cultura: } \\
\text { Asignaturas cuyo contenido } \\
\text { se enfoque en temas } \\
\text { culturales, étnicos, de } \\
\text { género, religión, filosofía, } \\
\text { entre otros, o de la identidad } \\
\text { propia de la institución } \\
\text { educativa. } \\
\text { 4. Interdisciplinariedad } \\
\text { otras áreas del } \\
\text { conocimiento: Formación } \\
\text { con enfoques distintos a las } \\
\text { ciencias económicas como } \\
\text { humanidades, ingeniería, } \\
\text { ciencias naturales, arte, } \\
\text { entre otras. } \\
\text { 5. Ética: Asignaturas } \\
\text { asociadas y derivadas de la } \\
\text { ética profesional. }\end{array}$ \\
\hline
\end{tabular}

Fuente: Elaboración propia.

Posteriormente, se utilizó la clasificación regional de distritos y municipios determinada en la Ley 617 de 2000, la cual los divide en siete categorías presentadas en la Tabla 2, de acuerdo a su nivel de población y a sus ingresos corrientes. Se utilizó esta categorización con el fin de determinar grupos con características demográficas similares para así, identificar posteriormente la influencia de variables contextuales en el diseño de programas de formación contable. Asimismo, se utilizó la Resolución 400 del 2019 para establecer en qué categoría se encuentra cada municipio y distrito de Colombia con vigencia para el año 2020. 
Pulgarín. L., Sandoval, J. y Navarro, P. Formación contable en Colombia...

Tabla 2: Categorización de distritos y municipios

\begin{tabular}{lll}
\hline \multicolumn{1}{c}{ Categoría } & \multicolumn{1}{c}{ Población (en habitantes) } & \multicolumn{1}{c}{ Ingresos (en salarios mínimos legales mensuales) } \\
\hline Especial & Superior o igual a 500.001 & Superiores a 400.000 \\
Primera & Entre 100.001 y 500.000 & Superiores a 100.000 y hasta 400.000 \\
Segunda & Entre 50.001 y 100.000 & Superiores a 50.000 y hasta 100.000 \\
Tercera & Entre 30.001 y 50.000 & Superiores a 30.000 y hasta 50.000 \\
Cuarta & Entre 20.001 y 30.000 & Superiores a 25.000 y hasta 30.000 \\
Quinta & Entre 10.001 y 20.000 & Superiores a 15.000 y hasta 25.000 \\
Sexta & Igual o inferior a 10.000 & No superiores a 15.000 \\
\hline
\end{tabular}

Fuente: Elaboración propia con base en la Ley 617 de 2000 (Congreso de la República de Colombia,

2000, art. 2).

Dado que en Colombia hay 1101 municipios $^{1}$ y 339 programas activos de formación superior en el área contable distribuidos entre técnicos, tecnólogos y profesionales ${ }^{2}$, se hizo uso de un muestreo aleatorio simple con el fin de facilitar la recolección de información y la interpretación de los resultados. Para la selección del tamaño de la muestra, se tomó un nivel de confianza del $95 \%$, un margen de error del $5 \%$ y una proporción esperada de 0,5 ; arrojando una muestra de 285 municipios, distribuyendo esta cantidad proporcionalmente de acuerdo a los pesos relativos de la composición municipal de cada categoría en Colombia y corrigiendo la categoría especial para incluir la totalidad de la población y las capitales de los 32 departamentos dado su nivel de importancia en la concentración poblacional e ingresos.

Se procedió a buscar en las bases de datos del SNIES cuántos y cuáles son los programas activos de formación técnica, tecnológica y profesional en el área contable. Se encontró que el 90,86\% del total de programas a nivel nacional, se encuentra en los 285 municipios seleccionados, es decir un total de 308.

De estos 308 se seleccionó una muestra no probabilística de 100 programas que representan a los municipios seleccionados. Para dichos programas, se hizo una revisión de los planes de estudio identificando si en ellos se evidencia influencia de las variables determinadas en la primera parte de este diseño muestral, observando si se hace alusión explícita en el plan de estudios.

1 Categorizados como tal por la Resolución 400 del 2019 de la CGN para la vigencia 2020 (por la cual se expide la certificación de categorización de las entidades territoriales).

2 Para que la información fuese más precisa se hizo análisis únicamente a nivel de pregrado; sin embargo, se tomaron los tres niveles de formación (técnico, tecnólogo y profesional) dado que, en el país, la oferta académica en el área contable se concentra fuertemente en los tres niveles, por tanto, no conviene descartar ninguno. 
Asimismo, se revisó otra información como la misión y visión del programa y los perfiles de ingreso, del estudiante y del egresado para identificar si las variables también son parte fundamental del programa en sí mismo.

En la medida en que se contaba con quince variables contextuales (cinco por cada componente) se le otorgó un punto a cada una. De este modo, por cada variable que se evidenciara explícitamente en un plan de estudios, un perfil de ingreso o egreso, en la misión o en la visión, se otorgaba un punto a cada programa y posteriormente se promediaba con los demás programas del mismo nivel de estudios en la misma categoría distrital o municipal.

Finalmente, se procedió a tabular los puntajes obtenidos por categoría y por nivel de formación para, de este modo, llevar a cabo un análisis cuantitativo de los mismos mediante gráficas de radar que permitieran determinar cuál de los tres niveles de formación se veía influenciado en mayor o menor medida por cada tipo de contexto en las distintas categorías de estudio.

\section{Resultados}

Gráfica 1: Influencia del contexto en la categoría 1

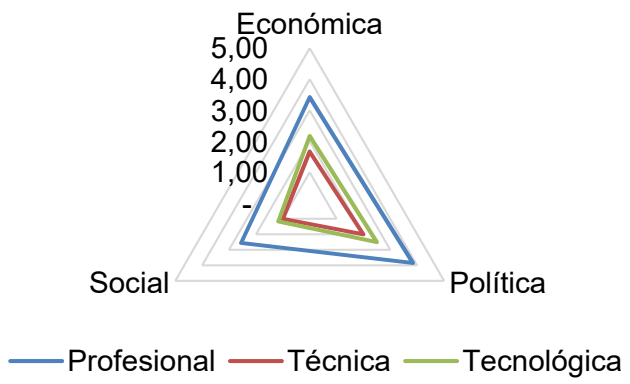

Fuente: Elaboración propia.

En la Gráfica 1 se observa que en el nivel de formación profesional es en donde más se presenta influencia de los contextos social, político y cultural en los programas curriculares del área contable ofertados en los municipios de la categoría 1. Dicha influencia, es mucho más evidente en el ámbito político, donde el promedio que se obtuvo de puntaje sobre 5.0 fue de 3.8, mientras que en el económico y el social se obtuvo 3.4 y 2.6 respectivamente. Por su parte, los niveles de formación técnica y tecnológica, si bien tienen también influencia evidente del contexto, los puntajes en los tres ámbitos no superan 2.5. 
Pulgarín. L., Sandoval, J. y Navarro, P. Formación contable en Colombia...

Gráfica 2: Influencia del contexto en la categoría 2

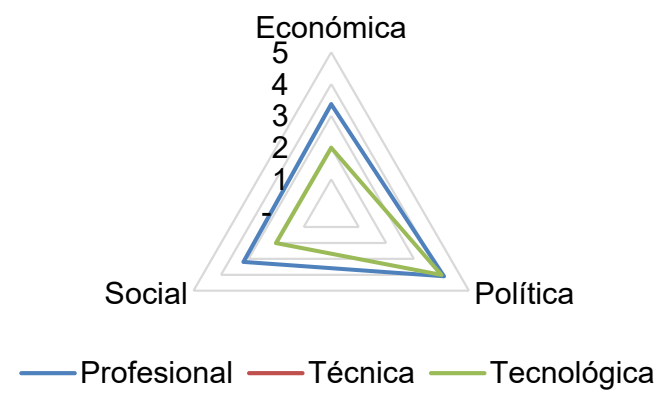

Fuente: Elaboración propia.

En la Gráfica 2 se observa nuevamente que en el nivel de formación profesional es en donde más se presenta influencia de los contextos social, político y cultural en los programas curriculares del área contable ofertados en los municipios de la categoría 2. Sin embargo, los hallazgos fueron particulares en esta categoría dado que, primero, el contexto político se ubicó en un puntaje de 4.0 tanto para el nivel profesional como para el tecnológico, indicando que la influencia de este aspecto es la misma para ambos procesos formativos; y segundo, no se oferta ningún programa técnico del área contable en los municipios que se encuentran en esta categoría.

Gráfica 3: Influencia del contexto en la categoría 3

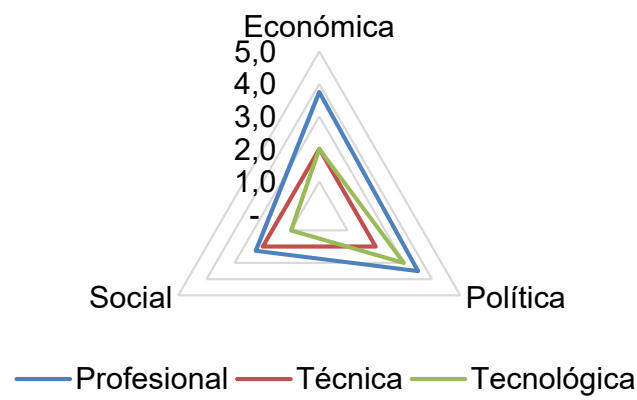

Fuente: Elaboración propia.

En la Gráfica 3 se observa que, de nuevo, el nivel de formación profesional es el que más se ve influenciado por el contexto, siendo las variables políticas y económicas aquellas con mayor afectación, con promedios de 3.5 y 3.8 respectivamente. Por su parte, el nivel tecnológico se ve altamente influenciado en el ámbito político en comparación al económico y al social, con puntajes de 3.0, 2.0 y 1.0 
respectivamente; mientras que en el nivel técnico los tres aspectos tienen una influencia similar, puntuando 2.0 en los tres casos.

Gráfica 4: Influencia del contexto en la categoría 4

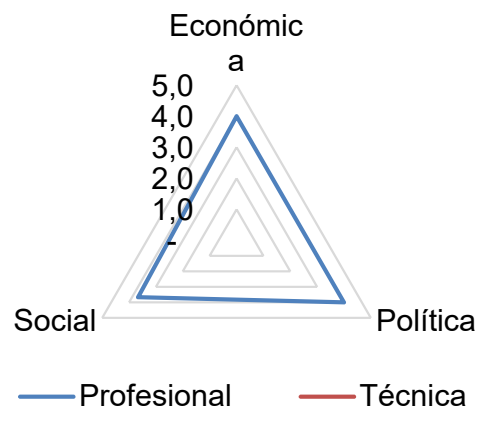

Fuente: Elaboración propia.

En la Gráfica 4, se observan las puntuaciones encontradas en los municipios de la categoría 4. En esta categoría, se encontró que ningún municipio oferta programas contables técnicos y tecnólogos; únicamente se ofertan a nivel profesional, donde la influencia promedio de los tres ámbitos es similar, puntuando alrededor de 4.0.

Gráfica 5: Influencia del contexto en la categoría 5

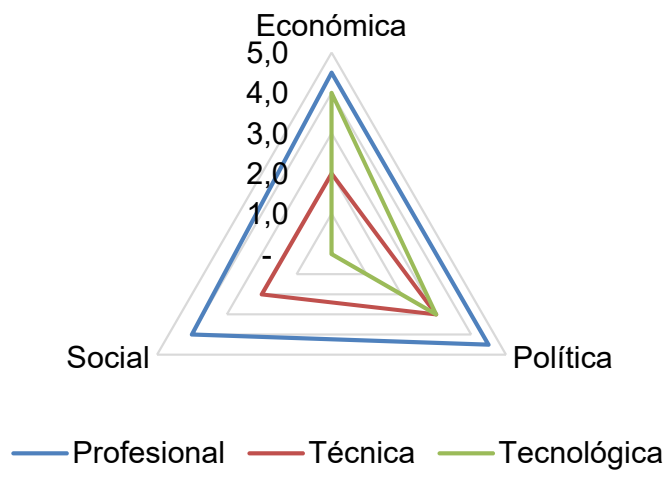

Fuente: Elaboración propia.

En la Gráfica 5 se tabularon los resultados obtenidos para la categoría 5. Nuevamente el nivel de formación profesional es el que más se ve influenciado por el contexto, sin embargo, la influencia de los tres ámbitos contextuales es mayor para esta categoría en comparación a las demás, superando en todos los casos el promedio de 4.0. Por su parte, se observa que la formación tecnológica se ve altamente influenciada por 
el ámbito económico y el político, no obstante, la influencia del ámbito social es nula. En cuanto a la formación técnica, la influencia es similar en los tres aspectos, siendo el político aquel que se destaca.

Gráfica 6: Influencia del contexto en la categoría 6

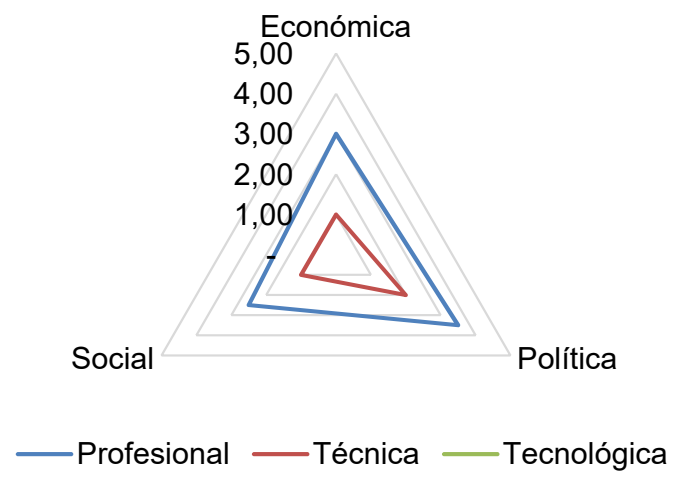

Fuente: Elaboración propia.

En la Gráfica 6 se observa de nuevo mayor influencia contextual en la formación profesional, siendo la política la de mayor puntaje $(3,5)$ y la social la de menor $(2,5)$. Por otro lado, es evidente que la formación técnica tiene muy poca influencia del contexto, dado que las variables económicas y sociales no superan en promedio el puntaje de 1.0 y la política el de 2.0. Adicionalmente, no se encontró oferta de programas tecnológicos en los municipios que se agrupan en esta categoría.

Gráfica 7: Influencia del contexto en la categoría especial

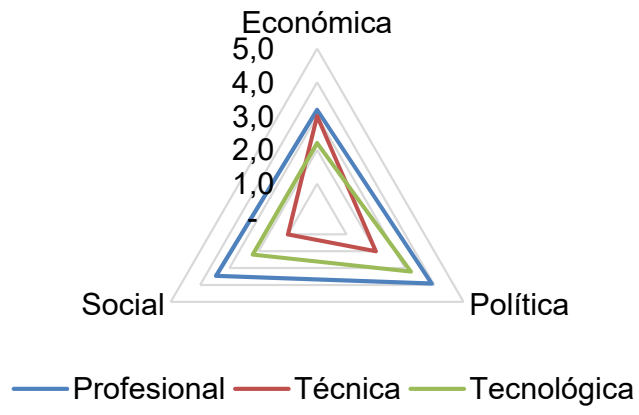

Fuente: Elaboración propia.

En la gráfica 7 , se tabularon los resultados para la categoría especial, donde al igual que en el resto de las categorías, el nivel de formación profesional es el que más afectación contextual presenta. Además, se puede ver una alta 
influencia del aspecto económico en la formación técnica y del político en la tecnológica, con puntajes de 3.0 y 3.2 respectivamente. Los demás puntajes no difieren significativamente de los resultados de las demás categorías.

\section{VI.Análisis y discusión}

En principio, el objetivo de este estudio consistió en analizar la formación de la disciplina contable en Colombia, bajo su contexto económico, político y social, a través de una revisión de las mallas curriculares, perfiles de ingreso, perfiles de egreso, misión y visión de los programas de educación superior en los niveles de técnico, tecnólogo y profesional, para contrastar los contenidos institucionales con los entornos previamente mencionados. Respecto a este objetivo se pudo encontrar que, en general, el contexto político es el que más influye en los procesos de diseño y difusión de currículos y planes de estudio en materia contable, especialmente en los programas de formación profesional. Aspectos asociados a las tecnologías, el sector público, la conservación del medio ambiente, la investigación e innovación y la presencia del derecho son fuertemente visibles en todos los municipios cotejados. En este sentido, se hace evidente que las políticas que el Estado ha impuesto relacionadas con cada uno de los aspectos mencionados acá han influenciado fuertemente a la formación superior y se manifiestan en la transformación de los programas, sus asignaturas y sus perfiles para adaptarse a estos requerimientos.

Adicionalmente, también es fuertemente visible la influencia del ámbito económico, más aún, dado el entorno globalizado y la normalización contable que han primado en las últimas décadas. Particularmente, se pudo observar que muchos programas de formación adecúan sus saberes a aquellos requeridos por el mercado laboral en materia de comercio exterior, libre mercado e IFRS. No obstante, también se encontró que existen programas en algunos municipios los cuales ofertan espacios académicos que responden a necesidades particulares del contexto local. Por ejemplo, hay una importante oferta de contenidos de contabilidades especiales y formación sectorial, orientadas a las actividades económicas regionales como el agro, la industria y los servicios financieros.

Por su parte, el contexto social es el que menos se percibe en los procesos de formación del área contable. Pese a que la mayoría de los programas profesionales incluyen espacios académicos de ética y responsabilidad social, estos no son tan comunes en los programas técnicos y tecnológicos. Por su parte, es escasa la inclusión de aspectos relativos a la identidad, la cultura y la interdisciplinariedad con áreas de conocimiento ajenas a las ciencias económicas. En este sentido, se encontraron muy pocos casos específicos de instituciones que incluyen en la formación contable cátedras de paz, democracia, territorio, artes, teatro, deporte y recreación. 
Pulgarín. L., Sandoval, J. y Navarro, P. Formación contable en Colombia...

Estos hallazgos son coherentes con las características específicas de calidad para los programas de formación profesional de pregrado en Contaduría Pública definidas por el MEN a través de la Resolución 3459 de 2003, según la cual el plan de estudios básico debe contener como mínimo cursos en los componentes del área de formación profesional de Ciencias contables y financieras, de formación organizacional, de información y de regulación, lo cual implica que las directrices establecidas por el gobierno nacional son determinantes en la formación de la disciplina contable en Colombia, al contrastar los contenidos institucionales con los entornos económico y político. En contraste, para el área sociohumanística propuesta por el MEN y directamente relacionada con el contexto social definido previamente en este trabajo, en componentes como la formación integral del Contador Público, la interdisciplinariedad y la ética profesional, no se evidencia esta lógica de cumplimiento normativo presente en los otros dos contextos.

Examinando particularmente los resultados para los programas técnicos y tecnológicos, se esperaría que por factores como por ejemplo el tiempo de duración y los temas específicos de la formación básica y profesional, se le dé una menor importancia al componente social; sin embargo, al revisar las características específicas de calidad para estos programas de formación en el área de la Administración definidas por el MEN, el área sociohumanística también debe contemplarse en los currículos, ya sea mediante cursos de responsabilidad social, ética o de contenido interdisciplinar.

Finalmente, en relación con estudios de naturaleza similar realizados previamente se establece que aun cuando se tratan temáticas cuyo objeto de estudio es la relación entre componentes de la configuración social y la formación universitaria, se orientan a contrastar las habilidades técnicas y conocimientos frente al mercado laboral (Del Cortea y Martínez, 2018; Rangland y Ramachandran, 2014; Setianto y Harahap, 2017), o si bien han estudiado elementos de los programas educativos (Loaiza, 2013; Patiño y Santos, 2009; Rojas y Sánchez, 2016; Valderrama et al., 2009; Valero, Patiño y Duque, 2013) se han enfocado exclusivamente en el pregrado universitario y sobre un reducido grupo de instituciones. Por lo que este trabajo propone un enfoque novedoso al abordar con mayor amplitud el estudio de los programas de formación contable en el país.

\section{Conclusiones}

Estos hallazgos son paradójicos en la medida en que, contextualmente hablando, ninguno de los tres aspectos debería tener mayor impacto sobre la educación contable en comparación con los demás. En términos de formación, "la educación profesional responde a las necesidades del entorno, no solo a las correspondientes a las empresas, sino a las necesidades sociales en general" 
(Patiño y Vásquez, 2013, p. 1026). Es necesario entonces reconocer que la disciplina contable se debe construir desde las múltiples aristas de la sociedad. En este sentido, Rueda (2011) argumenta que "históricamente la contabilidad parece tener un papel instrumental en la aplicación de políticas económicas y empresariales y un rol inexistente cuando las mismas políticas incluyen asuntos sociales" (p. 160).

La Universidad no debe ser sólo un espacio que ofrece el conocimiento instrumental que da al estudiante el boleto de entrada a la empresa. La Universidad debe proveer a los estudiantes de una formación que les permita entender el pasado y el presente y desde ahí recrear y potencializar las culturas regionales y los puentes de encuentro entre ellas. Dicho en otras palabras, la ciudad y el campo colombianos, deben problematizarse en la Universidad para desde ahí proponer el fortalecimiento y la aparición de las prácticas sociales que mantengan las identidades regionales que se han convertido en símbolos de defensa de la nación colombiana. (Rojas, 2008, p. 265)

Aunque es importante formar profesionales en las diversas ramas contables con un enfoque de mercado, dado que el principal campo de acción es la empresa, no se debe limitar únicamente a la economía, a las finanzas y a la concepción de la contaduría como un oficio técnico basado en principios establecidos por organismos internacionales que unifiquen los criterios de presentación de la información en los diferentes países. Por el contrario, es indispensable reconocer las diferencias económicas, políticas y sociales de los contextos, de manera que responda a las necesidades de cada ente territorial.

Adicionalmente se observó que las instituciones de educación superior en la determinación de sus planes de estudio dan mayor importancia a la inclusión de cursos enfocados en los componentes de formación básica y formación profesional, dejando a un lado la formación sociohumanística que se relaciona directamente con el contexto social, especialmente en los niveles técnico y tecnológico, a pesar de que también hace parte de las características específicas de calidad de los programas que el MEN ha determinado. Por lo anterior, no puede concluirse que la conformación de los currículos se encuentre del todo influenciada por el cumplimiento normativo, sino que la realidad política, económica y social, juegan un papel importante en las temáticas que debe incluir el plan de estudios. Sin embargo, vale la pena cuestionarse sobre las razones por las cuales el contexto social presenta una menor influencia en comparación con el político y el económico, por lo cual se deja abierta la posibilidad de profundizar en este tema de investigación.

Por otro lado, surgen también interrogantes sobre la ausencia de programas de formación de nivel técnico y tecnológico en tres de las categorías municipales observadas. No es claro si dicha ausencia se debe a una falta de oferta o de demanda de dichos programas a nivel local; sin embargo, lo cierto es que, de tratarse de una falta de oferta, esta situación influiría fuertemente 
Pulgarín. L., Sandoval, J. y Navarro, P. Formación contable en Colombia...

en procesos de movilidad y crecimiento regional. Esto deriva otros problemas de tipo económico, político y social que pueden ser objeto de estudio para investigaciones futuras.

\section{Referencias bibliográficas}

Aguilera, R. (2014). Complejidades impredecibles: desafíos de las Ciencias Sociales en el mundo contemporáneo. Estudios Políticos (31), 129-146. Recuperado de: http://www. scielo.org.mx/scielo.php?script=sci_arttext\&pid =S0185-16162014000100007

Asociación Colombiana de las Micro, Pequeñas y Medianas Empresas - ACOPI. (2019). Memorias del 64 Congreso Nacional MIPYME de ACOPI. CAJACOPI - Unidad Prado Barranquilla, Colombia. Recuperado de: https://acopi.org.co/memorias-64-congresonacional-de-la-mipyme-2019-2-2/

Bonilla, R. (2011). Apertura y reprimarización de la economía colombiana. Revista Nueva Sociedad, 231, 46-65. Recuperado de: https:/www.nuso.org/media/articles/ downloads/3752_1.pdf

Casanova, F. (2003). Formación profesional y relaciones laborales. OIT: Boletín Cinterfor No. 153. Recuperado de: https:/www.oitcinterfor.org/livedrupal/sites/default/files/file publicacion/casan.pdf

Colmenares de Carmonas, Loyda, Valderrama, Yosman, Perdomo Ramírez, Anggilyna, y Ramírez, Johan (2016). El proceso cognoscitivo de los estudiantes de Contaduría Pública de la ULA-NURR y su vinculación con la Deontología Contable. Educere, 20(65), 45-59. ISSN: 1316-4910. Recuperado de: https://www.redalyc.org/articulo. oa?id=356/35646429006

Confecámaras. (2018). Informe de dinámica empresarial en Colombia. Recuperado de: https:// redvalorcompartido.com/TEXTOS/TEXTOS2/Informe\%20de\%20din\%C3\%A1 mica\%20 empresarial\%20en\%20Colombia.pdf

Congreso de la República de Colombia. (1992). Ley 30 de 1992: Por la cual se organiza el servicio público de la Educación Superior. Diario Oficial No. 40.700, de 29 de diciembre de 1992. Bogotá, Colombia.

Congreso de la República de Colombia. (2000). Ley 617 de 2000: Por la cual se reforma parcialmente la Ley 136 de 1994, el Decreto Extraordinario 1222 de 1986, se adiciona la Ley Orgánica de Presupuesto, el Decreto 1421 de 1993, se dictan otras normas tendientes a fortalecer la descentralización, y se dictan normas para la racionalización del gasto público nacional. Diario oficial AÑO CXXXVI. N. 44188. 9, OCTUBRE, 2000. Bogotá, Colombia.

Congreso de la República de Colombia. (2002). Ley 749 de 2002: Por la cual se organiza el servicio público de la educación superior en las modalidades de formación técnica profesional y tecnológica, y se dictan otras disposiciones. Diario Oficial No. 44.872, de 19 de julio de 2002. Bogotá, Colombia.

Congreso de la República de Colombia. (2009). Ley 1314 de 2009: Por la cual se regulan los principios y normas de contabilidad e información financiera y de aseguramiento de información aceptados en Colombia, se señalan las autoridades competentes, el procedimiento para su expedición y se determinan las entidades responsables de vigilar su cumplimiento. Diario oficial No. 47.409 de 13 de julio de 2009. Bogotá, Colombia. 
Contaduría General de la Nación. (2007). Resolución 354 del 2007: Por la cual se adopta el Régimen de Contabilidad Pública, se establece su conformación y se define el ámbito de aplicación. Diario oficial 46.751 de septiembre 14 de 2007. Bogotá, Colombia.

Contaduría General de la Nación. (2014). Resolución 414 del 2014: Por la cual se incorpora, en el Régimen de Contabilidad Pública, el marco normativo aplicable para algunas empresas sujetas a su ámbito y se dictan otras disposiciones. Bogotá, Colombia.

Contaduría General de la Nación. (2015). Resolución 533 del 2015: Por la cual se incorpora, en el Régimen de Contabilidad Pública, el marco normativo aplicable a entidades de gobierno y se dictan otras disposiciones. Bogotá, Colombia.

Contaduría General de la Nación. (2017a). Resolución 037 del 2017: Por la cual se regula el Marco Normativo para Empresas que Cotizan en el Mercado de Valores, o que Captan o Administran Ahorro del Público. Bogotá, Colombia.

Contaduría General de la Nación. (2017b). Resolución 461 del 2017: Por la cual se incorpora, en el Régimen de Contabilidad Pública, el Marco Normativo para Entidades en Liquidación y se dictan otras disposiciones. Bogotá, Colombia.

Contaduría General de la Nación. (2019). Resolución 400 del 2019: Por la cual se expide la certificación de categorización de las entidades territoriales: departamentos, distritos y municipios, conforme a lo dispuesto en las Leyes 136 de 1994, 617 de 2000 y el Decreto 2106 de 2019. Bogotá, Colombia.

Cumbre Mundial sobre la Sociedad de la Información. (2005). Construir sociedades de la información que atiendan a las necesidades humanas. Revista de Estudios Sociales (22), 141-143. Recuperado de: https://www.redalyc.org/articulo.oa?id=815/81502214

Del Cortea, J. M., \& Martínez, G. M. F. (2018). Relevant competences in public accounting and finance: Is there a consensus among employers, professors and students? Contaduría y Administración, 63(2), 17-18. DOI: 10.22201/fca.24488410e.2018.1265

Departamento Administrativo de Ciencia, Tecnología e Innovación - Colciencias. (2018). Modelo de medición de grupos de investigación, desarrollo tecnológico o de innovación y de reconocimiento de investigadores del sistema nacional de ciencia, tecnología e innovación año 2018. Anexo 1. Bogotá D.C, Colombia.

Barragán-Días, D. M. (2008). Indagación sobre los discursos de curriculum en contaduría pública en Colombia. Revista Facultad de ciencias económicas: Investigación y reflexión, 16(2), 173-188. DOI: https://doi.org/10.18359/rfce.4477

Gómez, M. (2007). Comprendiendo las relaciones entre los sistemas contables, los modelos contables y los sistemas de información contables empresariales. Revista Legis Internacional de Contaduría y Auditoría, (32), 83-114. Recuperado de: https://www.researchgate.net/profile/Mauricio_Gomez6/publication/31609555_ Comprendiendo_las_relaciones_entre_los_sistemas_contables_los_modelos_ contables_y_los_Sistemas_de_informacion_contables_empresariales/ links/54df4eda0cf29666378966a3/Comprendiendo-las-relaciones-entre-lossistemas-contables-los-modelos-contables-y-los-Sistemas-de-informacion-contablesempresariales.pdf

Gómez Lee, M. I. (2008). Las políticas públicas en la sociedad globalizada. Revista Opera, (8), 175-194. Recuperado de: https://www.redalyc.org/articulo.oa?id=675/67500809 
Pulgarín. L., Sandoval, J. y Navarro, P. Formación contable en Colombia...

Jarne, José Ignacio. (1997). Clasificación y evolución internacional de los sistemas contables. Madrid: Ediciones AECA.

Jokinen, P. (2000). The information society: environmental policy perspective and beyond. Foresight, 2(2), 173-181. DOI: https://doi-org.ezproxy.unal.edu. co/10.1108/14636680010802627

León-Paime, E. F. (2008). Las facultades de la modernización: contexto de aparición de los programas de contaduría pública en Colombia. Revista de la Facultad de Ciencias Económicas: Investigación y Reflexión, 16(1), 41-58. DOI: https://doi.org/10.18359/ rfce.4482

León-Paime, E. F. (2009). La educación contable en el contexto anglosajón: una mirada a los años de construcción de comunidad. Cuadernos de contabilidad, 10(27). Recuperado de: https://revistas.javeriana.edu.co/index.php/cuacont/article/view/3208

Loaiza-Robles, F. (2013). Corrientes educativas internacionales presentes en programas de contaduría pública. Cuadernos de contabilidad, 14(34), 189-215. Recuperado de: https:// revistas.javeriana.edu.co/index.php/cuacont/article/view/6073

López, L. (2013). Estándares internacionales y educación contable. Apuntes del CENES, 239261. DOI: https://doi.org/10.19053/22565779.2075

Martínez Puón, Rafael. (2009). ¿Hacia dónde se dirige el campo de estudio de la administración pública? Convergencia, 16(49), 309-329. Recuperado de http://www. scielo.org.mx/scielo.php?script=sci_arttext\&pid=S1405-14352009000100013\&lng $=\mathrm{es} \& \mathrm{t} \operatorname{lng}=\mathrm{es}$

Mella, E. (2003). La educación en la sociedad del conocimiento y del riesgo. Revista Enfoques Educacionales, 5(1), 107-114. Recuperado de: https://revistas.uchile.cl/ index.php/REE/article/view/47517/49555

Ministerio de Educación Nacional. (2003a). Resolución 3459 de 2003. Por la cual se definen las características específicas de calidad para los programas de formación profesional de pregrado en Contaduría Pública. Bogotá, Colombia.

Ministerio de Educación Nacional. (2003b). Resolución 3462 de 2003. Por la cual se definen las características específicas de calidad para los programas de formación hasta el nivel profesional por ciclos propedéuticos en las áreas de las Ingeniería, Tecnología de la Información y Administración. Bogotá, Colombia.

Moreno, G. y Duque, Ó. (2016). La interdisciplinariedad en la formación contable: estudio del currículo en el programa de Contaduría Pública de la Universidad Santo Tomás, Bogotá. Revista Activos, 14(26), 25-60. Recuperado de https://revistas.usantotomas. edu.co/index.php/activos/article/view/3971

Navarro-Pérez, P. A., Pulgarín-García, L. N., y Sandoval-Alarcón, J. D. (2019). Formación de maestría en contabilidad en Colombia: una comparación entre las expectativas de los aspirantes y los contenidos curriculares de los programas. Revista Activos, 16(31), 157 203. DOI: https://doi.org/10.15332/25005278/5319

Observatory of Economic Complexity. (2018). Colombia: Complejidad económica, exportaciones e importaciones. Visualizaciones OEC. Recuperado de: https://oec. world/es/ 
Ortiz Bojacá, José Joaquín. (2009). Fronteras De La Investigación Contable-FinancieraEconómica: Autonomía E Interdisciplinariedad. Revista Facultad de Ciencias Económicas: Investigación y Reflexión, 17(1), 179-194. Recuperado de: http:/www.scielo.org.co/ scielo.php?script $=$ sci_arttext\&pid $=$ S0121-68052009000100012\&lng $=$ en\&tlng $=e s$.

Palacio-Mendoza, G., y Navas-Ríos, M. (2011). Las teorías curriculares en los programas contables: Caso Universidad de Cartagena. Panorama Económico, 19(19), 177-198.

Patiño, R., y Santos, G. (2009). La investigación formativa en los programas de Contaduría Pública, caso Colombia. Capic Review, (7), 23-34. Recuperado de: https://dialnet. unirioja.es/servlet/articulo?codigo $=3736534$

Patiño, R. y Vásquez, N. (2013). Las características de los subsistemas contables de Colombia y México ante la convergencia a Normas Internacionales de Información Financiera. Cuadernos de contabilidad, 14(36). Recuperado de: https://revistas.javeriana. edu.co/index.php/cuacont/article/view/7911

Peralta, L. y Vasco, M. (1998). El Estado y la Sociedad. Revista de Estudios Sociales, (2). Recuperado de: https://www.redalyc.org/articulo.oa?id=815/81511299018

Ragland, L., \& Ramachandran, U. (2014). Towards an understanding of excel functional skills needed for a career in public accounting: Perceptions from public accountants and accounting students. Journal of Accounting Education, 32(2), 113-129. DOI: https:// doi.org/10.1016/j.jaccedu.2014.03.002

Rojas Montoya, D. Y., y Sánchez Atehortúa, D. Y. (2016). Debilidades y fortalezas del plan de estudios del programa de contaduría pública de la Universidad de Antioquia (Seccional Oriente) respecto de las necesidades actuales de sus egresados. Contaduría Universidad De Antioquia, (69), 223-262. Recuperado de: https://revistas.udea.edu.co/ index.php/cont/article/view/328438

Rojas, W. R. (2008). Congoja por una educación contable fútil. Contaduría Universidad de Antioquia, (52), 259-274. Recuperado de: https://revistas.udea.edu.co/index.php/cont/ article/view/2171

Rojas, W. R., y Zapata, C. M. O. (2011). Consideraciones sobre el sentido de un proyecto educativo en Contaduría Pública. Cuadernos de administración, 27(45), 45-60. Recuperado de: https://www.redalyc.org/comocitar.oa?id=225019868004

Rueda, G. (2011). Contabilidad para la equidad y la inclusión social: propuestas para una investigación interdisciplinaria a largo plazo. Revista de la Facultad de Ciencias Económicas: Investigación y Reflexión, 19(1), 159-174. DOI: https://doi.org/10.18359/ rfce. 2266

Sebayang, M. M., \& Muda, I. (2020). To Be An Accountant-How Accounting Student Choose Their Career. Junior Scientific Researcher, 6(1), 34-40. Recuperado de: https://www.ceeol. com/search/article-detail id $=871694$

Setianto, A. I., \& Harahap, Y. A. (2017). Factors Affecting the Interests of Accounting Students Study Program Selection Career Public Accountants. Journal of Applied Managerial Accounting, 1(1), 51-61. DOI: https://doi.org/10.30871/jama.v1i1.1238

Tejeda González, J. L. (2014). Las dimensiones de la sociedad civil. Polis: Investigación y Análisis Sociopolítico y Psicosocial, 10(1), 133-156. Recuperado de: https://www.redalyc. org/articulo.oa?id=726/72631429006 
Pulgarín. L., Sandoval, J. y Navarro, P. Formación contable en Colombia...

Uvalle, R. (2014). La importancia de la ética en la formación de valor público. Estudios políticos (32), 59-81. Recuperado de: http://www.scielo.org.mx/scielo.php?pid=S0185$16162014000200004 \&$ script $=$ sci_arttext\&tlng $=$ en

Valderrama-Prieto, M. A., Rodríguez-Cástro, B., Arias-Bello, M. L., Bejarano, D. F., Garzón, H. A., Salgado-Castillo, J. A., y Sosa-Cardozo, J. M. (2009). El plan de estudios del programa de Contaduría Pública de la Pontificia Universidad Javeriana frente a los retos que genera la Ley 1314 de 2009. Cuadernos de Contabilidad, 10(26), 113-145. Recuperado de: https://revistas.javeriana.edu.co/index.php/cuacont/article/view/3221

Valero, G., Patiño, R., y Duque, O. (2013). Competencias para el programa de Contaduría Pública: una aproximación conceptual. Contaduría Universidad De Antioquia, (62), 1136. Recuperado de: https://revistas.udea.edu.co/index.php/cont/article/view/18910

Yassim, M. (2019). The wicked problem of social cohesion: moving ahead. Journal of Social Marketing, 9(4), 507-521. DOI: https://doi-org.ezproxy.unal.edu.co/10.1108/ JSOCM-12-2018-0162 